\title{
9 Mitglieder der AG Chronische Wunde
}

- Azvedo, Chadwick-Thomas

- Bode, Thilo

- Gäbel, Gabor

- Horn, Thomas

- Kipfmueller, Brigitte

- Lehmann, Sylvia

- Liebetrau, Michael

- Phan, Truong Quang Vu

- Riedel, Evelyn

- Schüning, Andrea

- Seltmann, Tobias

- Wutzler, Sebastian

Unterstützt durch:

- Donath, Katharina

- Leuwer, Rudolf 